American Journal of Infectious Diseases 3 (1): 51-61, 2007

ISSN 1553-6203

(C) 2007 Science Publications

\title{
Current Approaches for the Prevention of Surgical Site Infections
}

\author{
${ }^{1}$ Sander Florman and ${ }^{2}$ Ronald Lee Nichols \\ ${ }^{1}$ Tulane Abdominal Transplant Institute, Tulane University School of Medicine, New Orleans, Louisiana \\ ${ }^{2}$ Department of Surgery, Tulane University School of Medicine, New Orleans, Louisiana
}

\begin{abstract}
Surgical site infections (SSIs) are the most common type of nosocomial infection among surgical patients and are commonly caused by the patients' own microbial flora. The prevalence of SSI is a major concern because of the associated increase in the incidence of morbidity and mortality, length of hospitalization and cost of care for postoperative patients. Key factors that determine whether patients are at risk for developing SSI include the inherent potential contamination of the surgical site, the duration of the operation and the individual patient susceptibility. Preventive preoperative measures that can reduce the risk of SSIs include administration of antimicrobial prophylaxis, proper utilization of skin antiseptic agents for both the patient and the surgical team, proper patient preoperative hair removal and the policy of canceling elective procedures when remote skin, urinary or pulmonary infections occur. This paper will review the efficacy and safety of available antiseptic agents, as well as discuss patient-specific prevention strategies.
\end{abstract}

Key words: Surgical site infections, antisepsis, prevention

\section{INTRODUCTION}

Nosocomial infections are the eighth leading cause of death in the United States ${ }^{[1]}$. These infections may be caused by accidental violations of infection control protocols, exposure to nonsterile environmental objects or transmission of opportunistic pathogens within the normal course of events. The most common nosocomial infections among all hospitalized patients include urinary tract infections, surgical site infections (SSIs) and respiratory tract infections. SSIs are most often the result of microbial contamination that occurs during surgical procedures. According to the National Nosocomial Infections Surveillance (NNIS) System of the Centers for Disease Control and Prevention (CDC), SSIs account for 14 to $16 \%$ of all nosocomial infections and are the most common nosocomial infections among surgical patients, accounting for $38 \%$ of such infections ${ }^{[2]}$. Approximately 500,000 patients will acquire an SSI during the 44 million inpatient surgical procedures performed annually in the United States ${ }^{[3,4]}$.

The prevalence of SSIs is a major concern because they increase the incidence of morbidity and mortality, length of hospitalization and cost of care for postoperative patients ${ }^{[3,4]}$. In a comprehensive followup study of 225 matched pairs of patients with and without SSIs, infected patients were twice as likely to die compared with uninfected patients ( 7.8 vs $3.5 \%$, respectively ${ }^{[5]}$. The median length of hospitalization was 6.5 days longer for infected patients compared with uninfected patients and it resulted in an excess direct cost of over $\$ 3000$ per patient. Moreover, a greater number of infected patients required readmission to the hospital within 30 days of their initial discharge, adding to the costs directly attributable to the SSIs. Therefore, preventive protocols that significantly reduce the incidence of SSIs would result in a concomitant reduction in days of hospitalization and related additional costs.

Although patient characteristics, such as age, gender, poor nutritional status, presence of diabetes and obesity, are known to be related to the risk of developing an SSI, preventive measures can reduce the surgery-associated risks for infection ${ }^{[2]}$. Traditional methods of preventing SSIs include, but are not limited to, preoperative administration of antibiotic prophylaxis and effective skin antisepsis for both the patient and the surgical team. The objective of this review is to discuss the issues surrounding SSIs, including risk factors, causative microorganisms and key perioperative prevention strategies.

Definitions of surgical site infections: In order to accurately assess the prevalence and epidemiology of SSIs, it is imperative that monitoring protocols are consistent. A system established by collaboration

Corresponding Author: $\quad$ Sander Florman, Tulane Abdominal Transplant Institute, Tulane University School of Medicine, New Orleans, Louisiana 
between the National Academy of Sciences and the National Research Council in 1964 classifies surgical sites according to the extent and location of the wound $^{[2]}$. As a result, surgical wounds are traditionally categorized as class I through $\mathrm{IV}^{[6]}$. Class I surgical wounds are clean and uninfected and the respiratory, alimentary, genital, or urinary tract is not entered. Inflammation is not encountered during a class I procedure and the frequency of developing an SSI is low. Infections that do occur are usually caused by Staphylococcus aureus (S. aureus) from the patient's own microbial flora of the skin. The incidence of $S$. aureus infections is a growing concern with the emergence of virulent, antibiotic-resistant strains in the community setting ${ }^{[7]}$.

Surgical wounds designated as class II through IV involve different degrees of contamination ${ }^{[2]}$. During a class II, clean-contaminated surgical procedure, the respiratory, alimentary, genital, or urinary tract is entered under controlled conditions in the absence of an unusual contamination. Infections can be caused by endogenous or exogenous, aerobic or anaerobic bacteria, depending on the source. Class III, or contaminated surgical wounds are accidental wounds where there is a major breach in asepsis or leakage from the gastrointestinal tract causing contamination in the absence of a previously established infection. Dirtyinfected wounds are designated as class IV. These are primarily chronic wounds with devitalized tissue and the presence of foreign bodies or fecal contamination. Class IV wounds usually result from the presence of microorganisms in the surgical site prior to the procedure.

The CDC also recognized the importance of classifying and standardizing SSI definitions based on the anatomic location of the surgical site and associated pathophysiologic changes. The proposed definitions take into account clinical findings and results of laboratory-based tests, as well as the diagnoses of the operating surgeons ${ }^{[6,7]}$.

In 1988 , the CDC initially proposed definitions to refer to infections resulting from surgical procedures ${ }^{[6]}$. At that time, SSIs were referred to as surgical wound infections, a misleading term because "wound" specifically refers to the site of the incision from the skin to the soft tissues. Infections that directly result from surgical procedures that involve other areas of the body as well, such as organs or internal spaces that are manipulated during the operation, were termed surgical site infections in 1992 by the $\mathrm{CDC}^{[7]}$.

According to the current CDC standards, SSIs are divided into 2 groups in order to differentiate between infections of the actual site of the surgical incision and

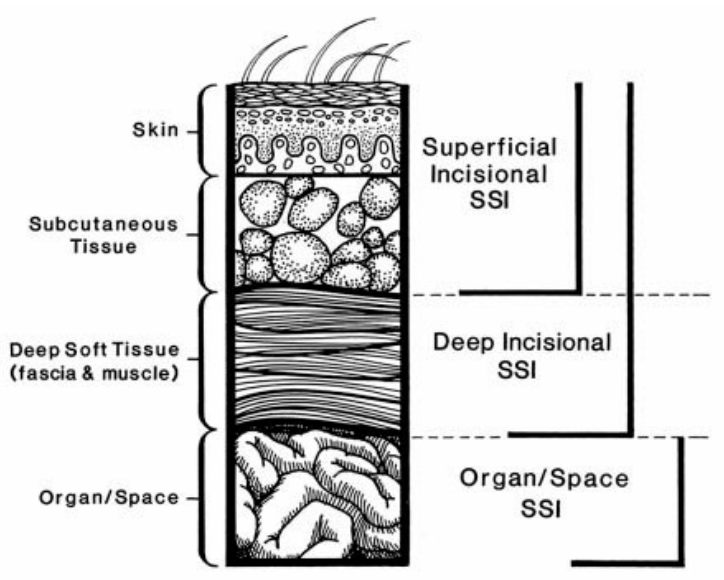

Fig. 1: Cross section depicting CDC classifications of SSI. Reprinted with permission from Mangram et al. ${ }^{[2]}$

infections of an anatomical part that was manipulated during the operation: incisional and organ/space, respectively (Fig. 1) ${ }^{[2,7]}$. Incisional SSIs are further classified as superficial or deep, depending on the anatomical part that is involved (Table 1) ${ }^{[2]}$. Superficial incisional SSIs involve the skin and the subcutaneous tissue, whereas deep incisional SSIs involve deep soft tissue layers, such as the fascial and muscle layers, of the incision.

In contrast, an organ/space SSI involves any part of the anatomy that was opened or manipulated during the surgical procedure. The specific organ or space involved is included as part of the definition to further identify the location of the infection. For example, if a patient had an appendectomy and subsequently developed an intra-abdominal abscess that was not directly associated with the incision, the infection would be reported as an organ/space SSI at the intraabdominal site. In order to be classified as an SSI, the infection must occur within 30 days of the surgical procedure or within 1 year if it is associated with a surgical implant and the infection appears to be related to the operation. Other parameters, such as purulent drainage from the incision, the isolation of the causative microorganism from the surgical site, or pain, localized swelling, redness and heat must also be present for the infection to be reported as an SSI (Table 1) ${ }^{[2]}$.

Microbiology of surgical site infections: Effective perioperative care is imperative for the prevention of SSIs and must address the microorganisms most likely to be encountered during the planned procedure. The pathogens responsible for SSIs vary depending on the type of surgery but are usually microorganisms found 
Am. J. Infect. Dis., 3 (1): 51-61, 2007

Table 1: Criteria for defining surgical site infections (SSIs) (adapted with permission from Mangram et al. ${ }^{[2]}$ )

Superficial incisional SSI
Anfection occurs within 30 days after operation
Infection involves only skin or subcutaneous tissue of the incision
and at least 1 of the following:
1. Purulent drainage from the superficial incision
2. Organisms isolated from the superficial incision
3. At least 1 of these signs or symptoms of infection: pain or tenderness, swelling, redness or heat and
superficial incision is deliberately opened by surgeon, unless incision is culture-negative
4. Diagnosis of superficial incisional SSI by the surgeon or physician
Infection occurs within 30 days after operation if no implant is left in place or within 1 year if implant is in
place and the infection appears to be related to the operation
and
Infection involves deep soft tissues of the incision
and at least 1 of the following:
1. Purulent drainage from the deep incision but not from the organ/space component of the surgical site
2. A deep incision spontaneously dehisces or is deliberately opened by a physician when the patient has at
least 1 of these signs or symptoms of infection: fever, localized pain or tenderness, unless the site is culture-
negative
3. An abscess or other evidence of infection involving the deep incision is found
4. Diagnosis of a deep incisional SSI by a surgeon or physician
Infection occurs within 30 days after operation if no implant is left in place or within 1 year if implant is in
place and the infection appears to be related to the operation
and
Infection involves any part of the anatomy, other than the incision, which was opened or manipulated during
an operation
and at least 1 of the following:
1. Purulent drainage from a drain that is placed through a stab wound into the organ/space
2. Organisms isolated from fluid or tissue in the organ/space
3. An abscess or other evidence of infection is found
4. Diagnosis or an organ/space SSI by a surgeon or physician

on or present within the surgical patient. For example, $S$. aureus from the patient's own skin microbial flora is the most common pathogen isolated from postoperative wound infections in clean surgical procedures (Class I).

In general, $S$. aureus, coagulase-negative staphylococci, Enterococcus species and Escherichia coli $(E$. coli) are the most frequently isolated pathogens in SSIs and the presence of each is determined primarily by the anatomical location and inherent contamination of the surgical $\operatorname{site}^{[2]}$. In cleancontaminated (Class II), contaminated (Class III) and dirty-infected (Class IV) surgical procedures, different risks are present for exposure to pathogens. The diverse endogenous microbial flora that normally inhabit the surgically violated organs are the microorganisms most frequently isolated from these postoperative surgical wounds. Gram-negative bacteria, including E. coli and anaerobic Bacteroides fragilis, predominate in many of these postoperative wound infections ${ }^{[8]}$.

A major concern associated with the prevention of SSIs is the emergence of antibiotic-resistant bacteria. An increase in antibiotic resistance has occurred specifically with vancomycin-resistant enterococci (VRE), methicillin-resistant S. aureus (MRSA), thirdgeneration cephalosporin-resistant E. coli and imipenem- and quinolone-resistant Pseudomonas aeruginos $^{[9]}$. In addition, the recent emergence of $S$. aureus species with a decreased sensitivity to vancomycin is alarming, especially because $S$. aureus infections are common among SSIs ${ }^{[10]}$.

Strategies for prevention of SSIs must include consideration of prevalent patterns of infection and antibiotic resistance at individual hospitals and the community. Effective protocols and antimicrobial agents should be employed against the most likely causative agents to reduce the incidence of SSIs.

Risk factors for SSI development: Risk factors for the development of an SSI correspond with wound, procedural and patient variables that are significantly associated with and act as valid predictors for the development of an infection after a surgical procedure (Table 2$)^{[2]}$. Certain patient characteristics are thought to increase the risk for an SSI. These include extremes of age, poor nutritional status, presence of diabetes, obesity, nicotine or steroid use, a coincident infection or colonization and a dysfunctional immune system ${ }^{[2]}$. Furthermore, external factors beyond the control of the patient, such as a prolonged preoperative stay that may increase the risk of exposure to opportunistic pathogens, also heighten the risk of developing an SSI. 
Am. J. Infect. Dis., 3 (1): 51-61, 2007

Table 2: Patient and operation risk factors for SSI (adapted with permission from Mangram et al. ${ }^{[2]}$ )

\begin{tabular}{l}
\hline Patient \\
\hline Age \\
Nutritional status \\
Diabetes \\
Smoking \\
Obesity \\
Steroids \\
Coexistent infections at a remote body site \\
Altered immune system \\
Length of preoperative stay \\
\hline Operation \\
\hline Duration of surgical scrub \\
Skin antisepsis \\
Preoperative shaving \\
Preoperative skin preparation \\
Duration of operation \\
Antimicrobial prophylaxis \\
Operating room ventilation \\
Wound class \\
Inadequate sterilization of instruments \\
Foreign matter in the surgical site \\
Surgical drains \\
Surgical techniques \\
\hline
\end{tabular}

In addition, wound and procedural characteristics, such as wound class and skin preparation techniques, have significant influence on the risk of developing SSIs. Individually, each factor can only partially assess the risk for infections. However, a comprehensive assessment of all risk factors on an individual patient basis is a valid predictor for SSI risk.

As previously discussed, traditional wound classifications are a reasonably effective method to predict the inherent risk of developing an SSI from a specific procedure. For example, although the risk of developing an infection from a clean, Class I surgical procedure is low, the risk progressively increases from a Class I surgery to a Class IV surgery. Generally, the wound classification method does not take into account the varying intrinsic patient risk factors within any wound class. Patients undergoing surgical procedures may exhibit a number of risk factors that make them more susceptible to infection by an exogenous pathogen than the wound classification might indicate.

Accordingly, in 1985 as part of the Study on the Efficacy of Nosocomial Infection Control (SENIC), Haley et al. devised an SSI risk assessment model that emphasized the importance of assessing patients on an individual basis ${ }^{[11]}$. Haley included both patient and procedural characteristics to develop a more comprehensive method for risk stratification. The risk for developing an SSI was heightened for patients undergoing Class III or Class IV surgical procedures, as well as for patients with $\geq 3$ diagnoses at the time of surgery. Abdominal procedures and surgical procedures that last $\geq 2$ hrs were additional risk factors for microbial contamination and resultant surgical site infections ${ }^{[11]}$.

In Haley's study of 59,352 surgical patients, the overall rate of SSIs progressively increased from $2.9 \%$ in Class I to $12.6 \%$ in Class IV procedures. However, the variables of abdominal operation, operation duration longer than $2 \mathrm{hrs}$ and 3 or more discharge diagnoses for the patient were associated with low, medium and high risk of SSI within each wound classification. For example, among the surgical patients undergoing a clean-contaminated (Class II) surgical procedure, 3.9\% of patients developed an SSI, but not all patients were at the same risk level for acquiring an infection. According to Haley's risk index, only $0.6 \%$ of patients identified as low risk developed an infection, whereas 2.8 and $26.1 \%$ of patients identified as medium and high risk, respectively, developed an infection. Notably, there were no patients identified as low-risk undergoing contaminated or dirty-contaminated surgical procedures ${ }^{[11]}$.

A surgical site risk system developed by the NNIS attempted to improve on the SENIC index in several ways. This system uses a dichotomization of the American Society of Anesthesiologists (ASA) score. An ASA score of 3, 4 or 5 is considered a risk factor. Another criterion considered in this system is whether the operation is contaminated or dirty-infected. This system also adjusts the cutoff duration of surgery for the type of procedure, rather than a $2 \mathrm{hr}$ cut point for all procedures. The duration indicative of an increased risk is extended for more extensive surgical procedures; e.g., coronary artery bypass grafts that last for more than $5 \mathrm{hrs}$ are at increased risk. The SSI rates (number of infections per 100 operations) for patients with NNIS scores of $0,1,2$ and 3 were 1.5, 2.9, 6.8 and 13.0, respectively ${ }^{[12]}$.

Studies of specific types of surgical procedures in any given anatomical area play a role in determining risk. For example, laparoscopic colon surgery is associated with a lower risk for developing an SSI compared with traditional open surgical techniques ${ }^{[13]}$.

Prevention strategies: The general health and wellbeing of surgical patients play a major role in the risk for developing an SSI. Although some risk factors cannot be modified, general and patient-specific prevention strategies, when implemented in a consistent and appropriate manner, can minimize the risk of such infections. In 1999, the Hospital Infection Control Practices Advisory Committee of the CDC provided updated guidelines for the implementation of 
prevention techniques based on clinical data to decrease the occurrence of SSIs ${ }^{[2,14]}$. The guidelines rank the prevention strategies into categories according to the level of recommendation for implementation.

Category IA prevention measures are strongly recommended for implementation and are supported by well-designed experimental, clinical or epidemiologic studies. The guidelines include the administration of antimicrobial prophylaxis (AMP) and adherence to regulations regarding patient hair removal, as well as identifying and treating remote infections before undertaking elective operations. Category IB prevention measures are strongly recommended for implementation and are supported by some experimental and clinical evidence. These measures include a variety of patient and surgical team preoperative antiseptic techniques, such as antiseptic showering and scrubbing. Proper intraoperative ventilation, disinfection and sterility of surgical instruments are also included in Category IB. Category II techniques are suggested for implementation but less crucial in preoperative procedures because of the lack of conclusive data to support their standard use for prevention of SSIs. Examples of Category II recommendations include preparation of the skin in concentric circles from the incision site and limitation of the preoperative stay in the hospital. Unresolved issues include techniques with insufficient evidence or no consensus with regard to prevention of SSIs, such as preoperative improvement in nutritional status, use of mupirocin in nares, hypothermia and the technique to enhance wound space oxygenation.
A number of important practices for prevention of SSIs are common to all surgical procedures and primarily focus on preoperative preparation practices $(\text { Table } 3)^{[2,4]}$.

Antimicrobial prophylaxis: The consistent and correct administration of AMP is the most effective method to reduce the risk of acquiring an SSI in indicated Class I (clean) or Class II (clean-contaminated) surgeries ${ }^{[2]}$. By definition, AMP is not indicated in Class III or IV surgeries, whose patients are infected and presumed to be already receiving therapeutic antimicrobial agents ${ }^{[2]}$. The goal of AMP is to reduce the risk of contamination to a level that cannot overwhelm the host defense mechanism. Approximately 80 to $90 \%$ of surgical patients receive some type of AMP, but the procedures for administering this therapy are inappropriate in 25 to $50 \%$ of cases. Optimal administration requires that the correct agent is used, that initiation occurs within 30 to $60 \mathrm{~min}$ of incision and that the duration of drug administration does not last beyond $24 \mathrm{hrs}$ after the end of the surgery to achieve the maximum benefit.

The cephalosporin cefazolin is the antibiotic of choice in most clean procedures and is cost-effective, safe and effective against a broad spectrum of bacteria $^{[15,16]}$. Prophylaxis should be administered within $1 \mathrm{hr}$ of initiation of the surgery to maintain an effective antibiotic serum concentration throughout the entire course of surgery ${ }^{[17]}$. Redosing of the AMP is required if surgery is delayed beyond $1 \mathrm{hr}$ after the administration of the AMP and if the

Table 3: Recommendations for preoperative procedures from the Hospital Infection Control Practices Advisory Committee ${ }^{[2]}$ (adapted with permission from Nichols et $\mathrm{al}^{[4]}$ )

\begin{tabular}{|c|c|}
\hline Ranking & Recommendations \\
\hline Category IA & $\begin{array}{ll}\text { - } & \text { Treat remote infection before elective operation } \\
\text { - } & \text { Administer antimicrobial prophylaxis as indicated } \\
\text { - } & \text { Do not remove hair from operative site unless necessary to facilitate surgery } \\
\text { - } & \text { If hair is removed, do so immediately before surgery, preferably with electric clippers }\end{array}$ \\
\hline Category IB & $\begin{array}{l}\text { - } \\
\text { - } \\
\text { - } \\
\text { - Utient should shower or bathe night before operative procedure with an antiseptic agent } \\
\text { - } \\
\text { Surgical team memberian incision site before performing antiseptic skin preparation } \\
\text { antiseptic agent } \\
\text { After scrub, keep hands up and away from body. Dry hands with a sterile towel and don sterile } \\
\text { gown and gloves } \\
\text { Cessation of tobacco use } 30 \text { days before surgery }\end{array}$ \\
\hline Category II & $\begin{array}{l}\text { - } \quad \text { Prepare skin in concentric circles from incision site } \\
\text { - } \quad \text { Keep preoperative stay in hospital as short as possible }\end{array}$ \\
\hline Unresolved & $\begin{array}{ll}\text { - } & \text { Improve nutritional status } \\
\text { - } & \text { Use of mupirocin in nares } \\
\text { - } & \text { Taper or discontinue systemic steroid use before elective surgery } \\
\text { - } & \text { Hypothermia } \\
\text { - } & \text { Hyperoxia }\end{array}$ \\
\hline
\end{tabular}


Am. J. Infect. Dis., 3 (1): 51-61, 2007

Table 4: Activity of antiseptic agents commonly used for preoperative skin preparation and surgical scrubs (adapted with permission from

\begin{tabular}{|c|c|c|c|c|c|c|c|c|c|}
\hline Agent & $\begin{array}{l}\text { Gram- } \\
\text { positive } \\
\text { bacteria }\end{array}$ & $\begin{array}{c}\text { Gram- } \\
\text { negative } \\
\text { bacteria }\end{array}$ & Mtb & Fungi & Virus & $\begin{array}{l}\text { Rapidity of } \\
\text { action }\end{array}$ & $\begin{array}{c}\text { Residual } \\
\text { activity }\end{array}$ & Toxicity & Uses \\
\hline Chlorhexidine & $\mathrm{E}$ & $\mathrm{G}$ & $\mathrm{P}$ & $\mathrm{F}$ & $\mathrm{G}$ & Intermediate & $\mathrm{E}$ & $\begin{array}{l}\text { Ototoxicity } \\
\text { Keratitis }\end{array}$ & SP SS \\
\hline Alcohol & $\mathrm{E}$ & $\mathrm{E}$ & G & G & G & Most rapid & None & $\begin{array}{l}\text { Drying } \\
\text { Volatile }\end{array}$ & $\begin{array}{l}\text { SP } \\
\text { SS }\end{array}$ \\
\hline Iodine/ iodophors & $\mathrm{E}$ & G & G & G & G & Intermediate & Minimal & $\begin{array}{l}\text { Absorption } \\
\text { Skin irritation }\end{array}$ & $\begin{array}{l}\text { SP } \\
\text { SS }\end{array}$ \\
\hline PCMX & G & $\mathrm{F}^{*}$ & $\mathrm{~F}$ & $\mathrm{~F}$ & $\mathrm{~F}$ & Intermediate & G & $\begin{array}{c}\text { More data } \\
\text { needed }\end{array}$ & SS \\
\hline Triclosan & G & G & G & $\mathrm{P}$ & $\mathrm{U}$ & Intermediate & E & $\begin{array}{c}\text { More data } \\
\text { needed }\end{array}$ & SS \\
\hline
\end{tabular}

$\mathrm{E}=$ excellent $; \mathrm{F}=$ fair $; \mathrm{G}=$ good $; \mathrm{Mtb}=$ Mycobacterium tuberculosis $; \mathrm{P}=$ poor; $\mathrm{PCMX}=$ parachlorometaxylenol; $\mathrm{SP}=$ skin preparation; $\mathrm{SS}=$ surgical scrub; $\mathrm{U}=$ unknown. *Fair, except for Pseudomonas spp; activity improved by addition of chelating agent such as EDTA.

duration of surgery is longer than a period that is determined by achievable tissue levels of the antibiotic, the serum half-life of the antibiotic and its efficacy against anticipated organisms ${ }^{[2]}$.

Although it has been a standard practice to administer AMP after surgery for as long as surgical implants are in place, data have indicated that this practice is not necessary and can be harmful ${ }^{[2,18]}$. The administration of AMP beyond $24 \mathrm{hrs}$ after surgery has been associated with the emergence of antibioticresistant microorganisms and is not recommended ${ }^{[19]}$.

Preoperative skin preparation: The preoperative skin preparation of both the patient and the surgical team are essential in the prevention of SSIs. The mainstays of these recommendations are preoperative showering and skin antisepsis of the patients and scrubbing for the surgical team. These recommendations are all classified as CDC category IB (i.e., strongly recommended and supported by some experimental and clinical evidence).

Preoperative showering: Because most SSIs are associated with skin flora, the CDC strongly recommends preoperative showering by the patient the day before surgery ${ }^{[2]}$. Showering with an antiseptic or detergent agent can significantly decrease skin microbial counts. Using chlorhexidine has been shown to reduce skin bacterial counts as much as 9-fold, whereas povidone-iodine and triclocarban soap reduced skin bacterial counts 1.3-fold and 1.9-fold, respectively ${ }^{[20]}$. Furthermore, the overall incidence of SSIs has been observed to be lower when chlorhexidine is used for preoperative showering $(9 \%)$ than for those patients that showered with bar soap or placebo (12.8 and $11.7 \%$, respectively) ${ }^{[21]}$. In these patients, the incidence of $S$. aureus infections in clean procedures was reduced by half in patients who showered with chlorhexidine compared with bar soap.
Skin antisepsis: Many agents are available for use as skin antiseptic agents and are, in general, combinations of iodophors, chlorhexidine gluconate (CHG), parachlorometaxylenol (PCMX) and alcohol (Table 4$)^{[2]}$. Understanding the mechanism of action of each of these agents is critical to choosing the best agent for antisepsis ${ }^{[22]}$. The antibacterial action of iodophors occurs by penetration of the bacterial cell wall, allowing iodine entry. The bacteriocidal activity of $\mathrm{CHG}$ occurs by disrupting the cytoplasmic membrane. PCMX exerts its effects by inactivating bacterial enzymes. Alcohol primarily denatures proteins. An optimal antiseptic agent must reduce the initial microbial count and have persistent inhibition of microbial growth during the course of the surgical procedure. In addition, the antimicrobial action should be effective in the presence of blood.

Multiple studies have shown that $\mathrm{CHG}$ and CHG/alcohol solutions display these important properties. CHG plus 70\% isopropyl alcohol (IPA) has demonstrated efficacy against a wide range of bacteria, including $P$. aeruginosa, $S$. aureus and antibioticresistant bacteria (Table 5) ${ }^{[23]}$. Furthermore, CHG/IPA exhibits a rapid onset of action, persists for up to $24 \mathrm{hrs}$ and has increased efficacy with repeated applications $^{[23,24]}$. Chlorhexidine is not inactivated in the presence of blood, which neutralizes the effects of iodine and PCMX and dilutes the effects of alcohol. The combination of these parameters makes CHGcontaining agents more effective than many other antiseptic agents at reducing skin and wound microbial counts and thus decreasing SSIs ${ }^{[25-27]}$.

There is also significant benefit to $\mathrm{CHG}$ in combination with soap; the surfactant can remove the bioburden, including spores, which are increasingly more problematic with clostridial infections. Alcohol is not effective against spore removal. Iodine-containing solutions with surfactants are also effective at removing 
Am. J. Infect. Dis., 3 (1): 51-61, 2007

Table 5: Time kill results of common antiseptic agents against various microorganisms (adapted from Paulson with permission ${ }^{[23]}$ )

\begin{tabular}{|c|c|c|c|c|}
\hline \multirow[b]{3}{*}{ Exposure time } & \multicolumn{4}{|c|}{$\log _{10}$ eduction from initial population } \\
\hline & \multicolumn{2}{|c|}{ Planktonic } & \multicolumn{2}{|l|}{ Biofilm } \\
\hline & $15 \mathrm{sec}$. & $2 \mathrm{~min}$. & $15 \mathrm{sec}$. & $2 \mathrm{~min}$. \\
\hline \multicolumn{5}{|l|}{ S. aureus } \\
\hline $2 \% \mathrm{CHG}+70 \% \mathrm{IPA}$ & $>6$ & $>6$ & 1.51 & $>6$ \\
\hline 7.5\%P-I+72\%IPA & $>6$ & $>6$ & 0.37 & $>5$ \\
\hline $0.25 \% \mathrm{ZP}+73 \% \mathrm{EtOH}$ & $>5$ & $>5$ & 0.10 & $>5$ \\
\hline $62 \% \mathrm{EtOH}+<5 \% \mathrm{IPA}$ & $>6$ & $>6$ & 0.08 & \\
\hline \multicolumn{5}{|l|}{ MRSA } \\
\hline $2 \% \mathrm{CHG}+70 \% \mathrm{IPA}$ & $>6$ & $>6$ & 3.14 & $>6$ \\
\hline $7.5 \% \mathrm{P}-\mathrm{I}+72 \% \mathrm{IPA}$ & $>6$ & $>6$ & 0.75 & $>6$ \\
\hline $62 \%$ EtOH $+<5 \%$ IPA & $>6$ & $>6$ & 0.08 & $>6$ \\
\hline \multicolumn{5}{|l|}{ S. epidermidis } \\
\hline $2 \% \mathrm{CHG}+70 \% \mathrm{IPA}$ & $>6$ & $>6$ & 1.86 & $>6$ \\
\hline $7.5 \% \mathrm{P}-\mathrm{I}+72 \% \mathrm{IPA}$ & $>6$ & $>6$ & 0.97 & $>6$ \\
\hline $0.25 \% \mathrm{ZP}+73 \% \mathrm{EtOH}$ & $>5$ & $>5$ & 0.28 & $>6$ \\
\hline $62 \% \mathrm{EtOH}+<5 \% \mathrm{IPA}$ & $>6$ & $>6$ & 0.01 & $>6$ \\
\hline \multicolumn{5}{|l|}{ MRSE } \\
\hline $7.5 \% \mathrm{P}-\mathrm{I}+72 \% \mathrm{IPA}$ & $>5$ & $>5$ & 1.70 & $>5$ \\
\hline $62 \% \mathrm{EtOH}+<5 \% \mathrm{IPA}$ & $>5$ & $>5$ & 0.36 & $>5$ \\
\hline \multicolumn{5}{|l|}{ VREF } \\
\hline $2 \% \mathrm{CHG}+70 \% \mathrm{IPA}$ & $>5$ & $>5$ & $>5$ & $>5$ \\
\hline
\end{tabular}

spores but do not maintain the persistent antisepsis activity of CHG-containing solutions and are, therefore, not as effective overall at preventing SSIs.

Because surgical procedures may be associated with unique risks for wound contamination and SSIs because of the bacterial milieu present, the efficacy of antiseptic agents requires testing separately in various procedures. For example, higher infection rates occur following orthopedic procedures compared with procedures involving other areas of the body because the foot is more susceptible to bacterial colonization ${ }^{[28]}$. In a study of 125 patients who underwent surgery of the foot and ankle, CHG/IPA demonstrated greater efficacy in reducing the microbial load compared with an iodine/alcohol solution and chloroxylenol $(P<$ $0.0001)^{[26]}$. Similarly, antisepsis with CHG was more effective than povidone-iodine in reducing the preoperative bacterial load in the operative field for vaginal hysterectomies (22 vs $63 \%$, respectively; $P=$ 0.003), procedures in which SSIs are common complications $^{[27]}$.

Antiseptic solutions have also been tested against antibiotic-resistant microorganisms, which are a prevalent problem in SSIs. It has been hypothesized that disinfectants are less effective against MRSA than methicillin-sensitive $S$. aureus (MSSA). However, in an in vitro study of skin disinfectants on MRSA, the bactericidal activity of a $0.5 \%$ chlorhexidine/80\% alcohol solution was more effective and more rapid at reducing bacterial counts against both MSSA and MRSA compared with $0.5 \% \mathrm{CHG}$ and $10 \%$ povidoneiodine $^{[29]}$. Similar efficacy results have been reported in other studies against both MRSA and VRE ${ }^{[30,31]}$.

Preoperative scrubbing: Members of the surgical team who are intimately associated with the surgical procedure also perform a surgical scrub prior to surgery with an antiseptic agent. All members of the surgical team are required to scrub their arms and hands for at least $5 \mathrm{~min}$ before the first procedure of the day and for 2 to $5 \mathrm{~min}$ between subsequent procedures. The surgical hand scrub should display efficient, broad-spectrum antimicrobial properties on intact skin and contain a nonirritating, fast-acting antimicrobial preparation ${ }^{[32]}$. There are insufficient data to conclusively state that preoperative scrubbing with an antiseptic agent rather than a non-antimicrobial soap is more effective at preventing SSIs ${ }^{[33]}$. However, at least 1 outbreak of SSIs reportedly occurred when a surgeon switched from using an antiseptic surgical scrub product to a nonantimicrobial product ${ }^{[33]}$.

Surgical scrub solutions containing $\mathrm{CHG}$ have greater antimicrobial activity compared with other antiseptics. CHG products demonstrate a significant immediate and persistent antimicrobial effect, giving them a theoretical advantage during long surgical 
Am. J. Infect. Dis., 3 (1): 51-61, 2007

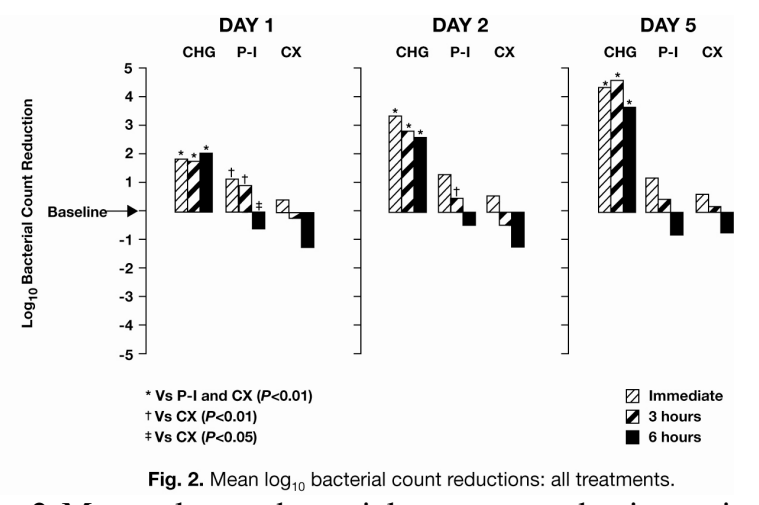

Fig. 2: Mean $\log _{10}$ bacterial count reductions in response to chlorhexidine gluconate $(\mathrm{CHG})$, povidone=iodine $(\mathrm{P}-\mathrm{I})$ and chloroxylenol $(\mathrm{CX})$ immediately after application and 3 and 6 hours later on days 1,2 and 5. Reprinted with permission from Aly and Maibach ${ }^{[35]}$

procedures ${ }^{[31,34]}$. In a study comparing scrubbing regimens of CHG/alcohol with povidone-iodine, the CHG regimen resulted in a $>50$-fold reduction in hand bacterial counts. In contrast, the povidone-iodine scrub reduced counts by more than 3 -fold ${ }^{[34]}$. In a similar study of 39 volunteers, scrubbing with $\mathrm{CHG} /$ alcohol solutions resulted in a significantly greater reduction in bacterial count compared with povidone-iodine and chloroxylenol scrubs at all sampling times, up to $6 \mathrm{hrs}$ after application $(P<0.01)$ (Fig. 2$)^{[35]}$.

Additional pre- and perioperative considerations: The 1999 recommendations of the Hospital Infections Control Practices Committee include several additional techniques to reduce the incidence of SSIs ${ }^{[2]}$.

Preoperative hair removal: The practice of shaving to remove hair from the surgical site the night before an operation is associated with a significantly higher risk for SSI compared with the use of a depilatory agent or no hair removal at all ${ }^{[2]}$. Therefore, it is recommended that hair removal be avoided. However, if hair removal is necessary, the use of clippers or depilatory agents is preferable over shaving to reduce the risk of surgical site contamination.

Pre- and perioperative glucose control: Inadequate control of glucose levels in surgical patients with diabetes is a predictive factor for the development of SSIs ${ }^{[36-38]}$. Patients with diabetes comprise between 17 and $20 \%$ of patients undergoing coronary artery bypass graftings ${ }^{[39]}$. The coronary artery bypass procedure is normally associated with an SSI rate of 0.8 to $2 \%$.
However, in patients with diabetes, the rate of SSI rises to $5.6 \%$ and is associated with increases in morbidity and treatment costs ${ }^{[39]}$. In addition, elevated blood glucose levels in the postoperative period are associated with an increased risk for developing an $\mathrm{SSI}^{[37]}$. Therefore, it is crucial to control blood glucose levels in patients with diabetes before they undergo surgical procedures and to avoid hyperglycemia during the procedures $^{[2]}$.

Preoperative tobacco cessation: Patients should be encouraged to abstain from cigarettes, cigars, pipes, or any other form of tobacco for at least 30 days before surgical procedures ${ }^{[2]}$. Current cigarette smoking has been implicated in increasing the incidence of SSIs following cardiac and other surgeries ${ }^{[40,41]}$.

Operative normothermia: Intraoperative hypothermia impairs immune function by causing vasoconstriction and a reduction in blood flow to surgical sites, which results in an increased susceptibility to $\mathrm{SSIs}^{[42]}$. It decreases the delivery of oxygen to tissues and inhibits the oxidative killing of pathogens by neutrophils. The normal core temperature can be maintained during surgery through the use of cotton blankets and active warming techniques, such as the use of warmed IV fluids and forced air warming. Although warmed IV fluids will not warm the patient, they effectively prevent the onset of hypothermia ${ }^{[43]}$.

In a clinical trial of 200 patients undergoing colorectal surgery, patients were randomized to either routine intraoperative thermal care (hypothermia) or additional warming procedures (normothermia) ${ }^{[44]}$. Warming techniques, which included the administration of warmed IV fluids and the use of a $40 \square \mathrm{C}$ forced-air cover, were effective in stabilizing body temperature, as the mean body temperature of patients in the normothermia group was higher compared with that of the patients in the hypothermia group $\left(36.6^{\circ} \pm 0.5^{\circ}\right.$ vs $34.7^{\circ} \pm 0.6^{\circ}$, respectively; $P<0.001$ ). Moreover, SSIs developed in only $6 \%$ of patients in the normothermia group compared with $19 \%$ of patients in the hypothermia group $(P=0.009)$. Also noted was an increase of 2.6 days in the mean duration of hospitalization for patients in the hypothermia group $(P$ $=0.001)$. Results from these studies indicate that maintaining normal body temperature throughout the surgical procedure appears to reduce the incidence of SSIs in surgical patients and decrease hospital stay. This promising technique should be utilized toady. The authors believe that this technique will be recommended when the authoritative committees next convene. 
Perioperative hyperoxia: Neutrophils use a method of oxidative killing to combat pathogens. This mechanism is compromised during surgical procedures because of a reduction in oxygen delivery to the tissues. All operations disrupt the vascular supply at the surgical site, causing the wound to become hypoxic compared with normal tissue ${ }^{[45]}$. Therefore, the supplemental administration of oxygen during surgical procedures may counterbalance the surgery-related hypoxia and reduces the risk for SSIs.

In a study of 300 patients undergoing colorectal surgery, patients were randomized to receive either 30 or $80 \%$ fraction of inspired oxygen intraoperatively and for $6 \mathrm{hrs}$ after the operation ${ }^{[46]}$. The higher oxygen level was associated with a 39\% reduction in the incidence of SSIs. Similar results were observed in a clinical trial of 500 patients undergoing colorectal resection who were randomized to receive either 30 or $80 \%$ inspired oxygen by sealed mask during and for $2 \mathrm{hrs}$ following the procedure ${ }^{[47]}$. SSIs developed in $11.2 \%$ of patients who received $30 \%$ inspired oxygen and in only $5.2 \%$ of patients who received $80 \%$ inspired oxygen. There are, however, other conflicting reports about the efficacy of perioperative hyperoxia. In a study of 165 patients undergoing major intra-abdominal procedures, patients were randomized to receive either 80 or $35 \%$ inspired oxygen and the results suggested a significantly higher SSI rate $(25 \%$ [20/80] vs $11 \%$ [9/80]) in those patients receiving $80 \%$ oxygen ${ }^{[48]}$. Because the cost of oxygen delivery is minimal compared with the increased costs incurred as a result of an SSI, perioperative oxygen administration is a practical method that may reduce the incidence of SSIs. Further studies of perioperative hyperoxia appear to be necessary before routine usage is universally recommended.

\section{CONCLUSION}

A comprehensive, multimodal approach is necessary for the effective prevention of SSIs, reflecting the complex etiology of these infections. Accepted methods for reducing the incidence and severity of SSIs, which include procedures for patients and healthcare providers, may be underutilized in many settings. Existing CDC guidelines for the use of antimicrobial prophylaxis, preoperative skin preparation for the patient and surgical team and perioperative practices provide evidence-based standards for effective preventive strategies. Implementation of CDC guidelines in all surgical and medical departments should be a priority, as there is significant potential to improve patient outcomes and to decrease hospital costs associated with preventable infections in surgical sites. Further clinical studies are needed to help optimize current practices and resolve unanswered questions about emerging strategies for the prevention of SSIs.

\section{REFERENCES}

1. Wenzel, R.P., 2002. Importance of infection control. In: Guide to Infection Control in the Hospital. 3rd Edn. (Eds. R.P. Wenzel, T.F. Brewer and J-P Butzler), pp: 1-4. B.C. Decker, Inc.

2. Mangram, A.J., T.C. Horan, M.L. Pearson, L.C. Silver and W.R. Jarvis, 1999. Guideline for prevention of surgical site infection, 1999. Hospital Infection Control Practices Advisory Committee. Infect. Control Hosp. Epidemiol., 20: 250-278.

3. DeFrances, C., M. Hall and M. Podgornik, 2005. 2003 National Hospital Discharge Survey. Adv Data, pp: 1-20.

4. Nichols, R.L., 2004. Current strategies for prevention of surgical site infections. Curr. Infect. Dis. Rep., 6: 426-434.

5. Kirkland, K.B., J.P. Briggs, S.L. Trivette, W.E. Wilkinson and D.J. Sexton, 1999. The impact of surgical-site infections in the 1990s: Attributable mortality, excess length of hospitalization and extra costs. Infect. Control Hosp. Epidemiol., 20: 725730 .

6. Garner, J.S., W.R. Jarvis, T.G. Emori, T.C. Horan and J.M. Hughes, 1988. CDC definitions for nosocomial infections. Am. J. Infect. Control, 16: 128-140.

7. Horan, T.C., R.P. Gaynes, W.J. Martone, W.R. Jarvis and T.G. Emori, 1992. CDC definitions of nosocomial surgical site infections, 1992: A modification of CDC definitions of surgical wound infections. Infect. Control Hosp. Epidemiol., 13: 606-608.

8. Nichols, R.L., 1991. Surgical wound infection. Am. J. Med., 91: 54S-64S.

9. Anonymous, 1999. National Nosocomial Infections Surveillance (NNIS) System Report, data summary from January 1990-May 1999, issued June 1999. Am. J. Infect. Control, 27: 520-532.

10. Rapp, R.P., 2000. Overview of resistant grampositive pathogens in the surgical patient. Surg. Infect. (Larchmt)., 1: 39-47.

11. Haley, R.W., D.H. Culver, W.M. Morgan, J.W. White, T.G. Emori and T.M. Hooton, 1985. Identifying patients at high risk of surgical wound infection. A simple multivariate index of patient susceptibility and wound contamination. Am. J. Epidemiol., 121: 206-215. 
12. Culver, D.H., T.C. Horan and R.P. Gaynes et al., 1991. Surgical wound infection rates by wound class, operative procedure and patient risk index. National Nosocomial Infections Surveillance System. Am. J. Med., 91: 152S-157S.

13. Gaynes, R.P., 2001. Surgical-site infections (SSI) and the NNIS Basic SSI Risk Index, Part II: Room for improvement. Infect. Control Hosp. Epidemiol., 22: 266-267.

14. Garner, J.S., 1993. The CDC Hospital Infection Control Practices Advisory Committee. Am. J. Infect. Control, 21: 160-162.

15. Page, C.P., J.M. Bohnen, J.R. Fletcher, A.T. McManus, J.S. Solomkin and D.H. Wittmann, 1993. Antimicrobial prophylaxis for surgical wounds. Guidelines for clinical care. Arch. Surg., 128: 79-88.

16. Anonymous, 2006. Antimicrobial prophylaxis for surgery. Treat. Guidel. Med. Lett., 4: 83-88.

17. Classen, D.C., R.S. Evans, S.L. Pestotnik, S.D. Horn, R.L. Menlove and J.P. Burke, 1992. The timing of prophylactic administration of antibiotics and the risk of surgical-wound infection. N. Engl. J. Med., 326: 281-286.

18. Kappstein, I. and F.D. Daschner, 1991. Use of perioperative antibiotic prophylaxis in selected surgical procedures--results of a survey in 889 surgical departments in German hospitals. Infection, 19: 391-394.

19. Hecker, M.T., D.C. Aron, N.P. Patel, M.K. Lehmann and C.J. Donskey, 2003. Unnecessary use of antimicrobials in hospitalized patients: current patterns of misuse with an emphasis on the antianaerobic spectrum of activity. Arch. Intern. Med., 163: 972-978.

20. Garibaldi, R.A., 1988. Prevention of intraoperative wound contamination with chlorhexidine shower and scrub. J. Hosp. Infect., 11 (Suppl. B): 5-9.

21. Hayek, L.J., J.M. Emerson and A.M. Gardner, 1987. A placebo-controlled trial of the effect of two preoperative baths or showers with chlorhexidine detergent on postoperative wound infection rates. J. Hosp. Infect., 10: 165-172.

22. Graves, P.B. and C.L. Twomey, 2006. Surgical hand antisepsis: An evidence-based review. Perioper. Nurs. Clin., 1: 235-249.

23. Paulson, D.S., 2005. Efficacy of preoperative antimicrobial skin preparation solutions on biofilm bacteria. AORN J., 81: 492-501; Quiz 503-506.

24. Hibbard, J.S., 2005. Analyses comparing the antimicrobial activity and safety of current antiseptic agents: A review. J. Infus. Nurs., 28: 194-207.
25. Mulberry, G., A.T. Snyder, J. Heilman, J. Pyrek and J. Stahl, 2001. Evaluation of a waterless, scrubless, chlorhexidine gluconate/ethanol surgical scrub for antimicrobial efficacy. Am. J. Infect. Control, 29: 377-382.

26. Ostrander, R.V., M.J. Botte and M.E. Brage, 2005. Efficacy of surgical preparation solutions in foot and ankle surgery. J. Bone Joint Surg. Am., 87: 980-985.

27. Culligan, P.J., K. Kubik, M. Murphy, L. Blackwell and J. Snyder, 2005. A randomized trial that compared povidone iodine and chlorhexidine as antiseptics for vaginal hysterectomy. Am. J. Obstet. Gynecol., 192: 422-425.

28. Miller, W.A., 1983. Postoperative wound infection in foot and ankle surgery. Foot Ankle, 4: 102-104.

29. Sakuragi, T., K. Yanagisawa and K. Dan, 1995. Bactericidal activity of skin disinfectants on methicillin-resistant Staphylococcus aureus. Anesth. Analg., 81: 555-558.

30. Frantz, S.W., K.A. Haines, C.G. Azar, J.I. Ward, S.M. Homan and R.B. Roberts, 1997. Chlorhexidine gluconate (CHG) activity against clinical isolates of vancomycin-resistant Enterococcus faecium (VREF) and the effects of moisturizing agents on $\mathrm{CHG}$ residue accumulation on the skin. J. Hosp. Infect., 37: 157-164.

31. Kampf, G., R. Jarosch and H. Ruden, 1998. Limited effectiveness of chlorhexidine based hand disinfectants against methicillin-resistant Staphylococcus aureus (MRSA). J. Hosp. Infect., 38: 297-303.

32. Paulson, D.S., 1994. Comparative evaluation of five surgical hand scrub preparations. AORN J., 60: 246, 249-256.

33. Boyce, J.M. and D. Pittet for the Healthcare Infection Control Practices Advisory Committee and the HICPAC/SHEA/APIC/IDSA Hand Hygiene Task Force, 2002. Guideline for hand hygiene in health-care settings. Recommendations of the Healthcare Infection Control Practices Advisory Committee and the HICPAC/SHEA/APIC/IDSA Hand Hygiene Task Force. Society for Healthcare Epidemiology of America/Association for Professionals in Infection Control/Infectious Diseases Society of America. MMWR Recomm. Rep., 51: 1-45; quiz CE1-4.

34. Grabsch, E.A., D.J. Mitchell, J. Hooper and J.D. Turnidge, 2004. In-use efficacy of a chlorhexidine in alcohol surgical rub: a comparative study. ANZ J. Surg., 74: 769-772. 
35. Aly, R. and H.I. Maibach, 1988. Comparative antibacterial efficacy of a 2 minute surgical scrub with chlorhexidine gluconate, povidone-iodine and chloroxylenol sponge-brushes. Am. J. Infect. Control, 16: 173-177.

36. Lilienfeld, D.E., D. Vlahov, J.H. Tenney and J.S. McLaughlin, 1988. Obesity and diabetes as risk factors for postoperative wound infections after cardiac surgery. Am. J. Infect. Control, 16: 3-6.

37. Zerr, K.J., A.P. Furnary, G.L. Grunkemeier, S. Bookin, V. Kanhere and A. Starr, 1997. Glucose control lowers the risk of wound infection in diabetics after open heart operations. Ann. Thorac. Surg., 63: 356-361.

38. Furnary, A.P., K.J. Zerr, G.L. Grunkemeier and A. Starr, 1999. Continuous intravenous insulin infusion reduces the incidence of deep sternal wound infection in diabetic patients after cardiac surgical procedures. Ann. Thorac. Surg., 67: 352360; Discussion, 360-362.

39. Golden, S.H., C. Peart-Vigilance, W.H. Kao and F.L. Brancati, 1999. Perioperative glycemic control and the risk of infectious complications in a cohort of adults with diabetes. Diab. Care, 22: 1408-1414.

40. Nagachinta, T., M. Stephens, B. Reitz and B.F. Polk, 1987. Risk factors for surgical-wound infection following cardiac surgery. J. Infect. Dis., 156: 967-973.

41. Jones, J.K. and R.G. Triplett, 1992. The relationship of cigarette smoking to impaired intraoral wound healing: a review of evidence and implications for patient care. J. Oral Maxillofac. Surg., 50: 237-239; Discussion, 239-240.
42. Ozaki, M., D.I. Sessler, H. Suzuki, K. Ozaki, C. Tsunoda and K. Atarashi, 1995. Nitrous oxide decreases the threshold for vasoconstriction less than sevoflurane or isoflurane. Anesth. Analg., 80: 1212-1216.

43. Sessler, D.I. and M. Schroeder, 1993. Heat loss in humans covered with cotton hospital blankets. Anesth. Analg., 77: 73-77.

44. Kurz, A., D.I. Sessler and R. Lenhardt, 1996. Perioperative normothermia to reduce the incidence of surgical-wound infection and shorten hospitalization. Study of Wound Infection and Temperature Group. N. Engl. J. Med., 334: 12091215.

45. Niinikoski, J., P. Jussila and T. Vihersaari, 1973. Radical mastectomy wound as a model for studies of human wound metabolism. Am. J. Surg., 126: 53-58.

46. Belda, F.J., L. Aguilera and J. Garcia de la Asuncion et al. for the Spanish Reduccion de la Tasa de Infeccion Quirurgica Group, 2005. Supplemental perioperative oxygen and the risk of surgical wound infection: a randomized controlled trial. JAMA, 294: 2035-2042.

47. Greif, R., O. Akca, E.P. Horn, A. Kurz and D.I. Sessler, 2000. Supplemental perioperative oxygen to reduce the incidence of surgical-wound infection. Outcomes Research Group. N. Engl. J. Med., 342: 161-167.

48. Pryor, K.O., T.J. Fahey, C.A. Lien and P.A. Goldstein, 2004. Surgical site infection and the routine use of perioperative hyperoxia in a general surgical population: A randomized controlled trial. JAMA, 291: 79-87. 